\title{
VALIDATION OF IMMUNOPHENOTYPING BY FLOW CYTOMETRY IN THE INVESTIGATION OF DIAGNOSTIC AND PROGNOSTIC MARKERS FOR BREAST CANCER
}

\author{
Daniella Serafin Couto Vieira', Sandro Wopereis ${ }^{1}$, Laura Otto Walter ${ }^{1}$, Maria Claudia Santos da Silva
}

'Universidade Federal de Santa Catarina - Florianópolis (SC), Brazil.

Introduction: Due to the high prevalence of breast cancer, it has a major financial impact on health systems. Currently, the diagnosis is made by morphological and immunohistochemical analysis (IHC). However, this methodology has some limitations. Therefore, methodologies capable of rapid and safe detection of tumor cells are needed, which can assist those already in use. Objectives: To validate immunophenotyping by flow cytometry (FC) in the investigation of diagnostic and prognostic markers for breast cancer; and to investigate lymphocytes subtypes infiltrated in the tumor and their relationship with tumor development. Methods: 52 breast tumor samples were sectioned and macerated in phosphatesaline buffer and stained with antibodies against estradiol (RE), progesterone (RP), HER2, Ki67, CD3, CD4, CD8 and CD45 receptors and analyzed by FC. All results were compared with IHC (reference method) in relation to sensitivity, specificity, positive predictive value (PPV) and negative predictive value (NPV), except for Ki67, where bias was compared between methodologies and correlation between lymphocyte subtypes and tumor characteristics. Results: The comparison of the FC with the IHC for each marker presented the RE analysis (sensitivity: 75\%, specificity: 90\%, PPV: 96.7\%, VPN: 47.4\%); PR analysis (sensitivity: 72\%, specificity: 70\%, PPV: 79.3\%, VPN: 60.8\%); analysis of HER2 (sensitivity: 80\%, specificity: 90.2\%, PPV: 66.7\%, VPN: 94.9\%). The analysis of Ki67 by FC was shown to be equivalent to IHC, with the advantage of not having an observational bias. No correlations were observed between the molecular subtype intratumor lymphocyte population profile and the tumor histological grade. Conclusion: The results show the FC's ability to safely and quickly detect breast cancer markers used in clinical practice. It is believed that the use of FC, in conjunction with morphological analysis and IHC, can overcome the individual limitations of each of the methodologies and provide reliable results in a faster and more efficient way, which will result in faster diagnoses and more accurate prognoses, directly benefiting patients. 